\title{
El profesorado quebequense frente a la «gestión basada en resultados». Un estudio cualitativo en cuatro Centros de Secundaria
}

\author{
Christian Maroy, Cécile Mathou, Samuel Vaillancourt ${ }^{1}$
}

\section{Resumen}

En el curso de los 15 últimos años, Quebec (Canadá) ha desarrollado una política de accountability -denominada Gestión Basada en Resultados (GBR)- que concierne a todo el conjunto del sector de la educación obligatoria de esta provincia ${ }^{2}$. Esta política se despliega en todos los niveles del sistema educativo (ministerio, comisiones escolares y centros educativos) y pone en práctica nuevas herramientas de planificación y de contractualización, así como también herramientas estadísticas que permiten hacer visibles los resultados (académicos) y establecer objetivos de rendimiento. La GBR implica una mirada de cambio de las prácticas del profesorado para hacerlas más «eficaces» y, así, contribuir a la realización de los objetivos de las políticas ministeriales: mejorar los índices de diplomados, las tasas de logro, reducir el abandono escolar. En este artículo analizamos las reacciones del profesorado de cuatro centros de secundaria a las demandas de cambio de las prácticas pedagógicas que les son indicadas por los gestores escolares. Para ello, nos basamos en una tipología de respuestas a las políticas educativas desarrollada por Cynthia Corbun (2004). Nuestro análisis muestra que las respuestas más frecuentes son el rechazo y el acomodo, pero también la renuncia a ciertas prácticas deseadas, así como las prácticas «imposibilitadas» por la puesta en marcha de la GBR, un tipo de respuesta que se agrega a la tipología de Corbun. Asimismo, mostramos que estas respuestas y sus significaciones varían según las prácticas establecidas por la organización institucional, pero también en función del contexto del centro educativo, y de las diversas estrategias y configuraciones de los actores implicados en ello.

\section{Palabras clave}

Accountability, políticas educativas, profesionalismo docente, gestión escolar, educación en Quebec (Canadá).

\footnotetext{
1 Universidad de Montreal, Cátedra de investigación de Canadá en políticas educativas. Este artículo se apoya en una investigación financiada por el Consejo de investigaciones en ciencias humanas (CRSH) de Canadá (n 435-012-0701).

2 En Canadá, la gestión administrativa territorial se basa en las llamadas provincias, que equivaldría a un estado dentro de un modelo federal.
} 


\section{Introducción}

La Gestión Basada en Resultados (GBR) es una política de responsabilización o de accountability en educación desarrollada desde el año 2000 por el gobierno de Quebec (Canadá). Inspirada en la nueva gestión pública (definición de objetivos por la autoridad política, flexibilidad de los medios para lograrlos, transparencia y evaluación, responsabilización y rendición de cuentas sobre los resultados), esta política es operacionalizada a través de las diversas herramientas de acción pública que van desde los planes estratégicos a los contratos, empleando al mismo tiempo las evaluaciones externas organizadas por el ministerio en sus principales filiales escolares. La definición de los objetivos es acompañada por el ajuste de indicadores a partir de las bases de datos estadísticas del ministerio, pero también de diversas herramientas para realizar el análisis y el seguimiento de la gestión de los centros educativos y de las comisiones escolares $(\mathrm{CE})^{3}$ que las supervisan.

Desde la Ley 88 (2008) que actualiza la GBR en la red de centros educativos públicos, las CE y los centros deben firmar «convenciones» con sus superiores (el ministerio o la CE) y comprometerse a lograr los objetivos calculados y definidos para contribuir a los cinco «objetivos» de la política ministerial: mejora de los porcentajes de diplomados en menos de 20 años, tasas de logro en las pruebas ministeriales en francés, reducción de las tasas de abandono escolar en el curso de la escolaridad, aumento del número de estudiantes en filiares profesionales, promoción de un ambiente sano y seguro en el centro educativo.

En nuestros trabajos anteriores, hemos mostrado que las CE se apoyan en la GBR para desplegar una nueva gestión de la pedagogía (Maroy et al., 2016; Maroy, 2017). Las CE persiguen extender el objeto y renovar las formas y las herramientas de la gestión escolar. Por una parte, actúan de manera más directa en el trabajo pedagógico del profesorado. Por otra parte, ellas se apoyan en una gama renovada de herramientas de gestión (herramientas estadísticas de análisis de resultados, herramientas de seguimiento de prácticas docentes) para orientar mejor el trabajo de las y los profesionales en el seno de las escuelas hacia el rendimiento esperado por los objetivos de la GBR. La GBR implica, en este sentido, una intención de seguimiento administrativo de las prácticas de las escuelas y de la «regulación», incluso, del cambio de las prácticas del profesorado para hacerlas más «eficaces», con la finalidad de contribuir a alcanzar los objetivos de rendimiento vehiculados por este modelo de gestión educativa.

En este artículo, analizamos las reacciones del profesorado de secundaria frente a las peticiones de cambio de las prácticas que les son solicitadas por los gestores escolares durante la puesta en marcha de la GBR. Apoyándonos en los trabajos de Cynthia Coburn (2004), nos interrogamos sobre la manera en que el profesorado responde a las demandas y presiones institucionales diversas, que provienen directamente de su equipo de dirección, pero también indirectamente de su CE.

Presentaremos, en una primera sección, nuestro marco teórico y metodológico. En segunda instancia, presentaremos las transformaciones de las prácticas docentes deseadas por la dirección de los centros de secundaria de la investigación. En una tercera sección, mostraremos las respuestas del profesorado, insistiendo en las tendencias comunes. Finalmente, discutiremos las fuentes de las variaciones entre los centros educativos analizados, derivadas de los contextos (población estudiantil, situaciones socioespaciales), de las estrategias de su dirección, o de la configuración de los actores y de sus relaciones.

\footnotetext{
3 Las comisiones escolares son entidades de regulación intermedia que están encargadas de poner en marcha en un territorio dado la educación pública. Organizan y guían los centros Primaria y Secundaria y los centros de formación profesional y formación de adultos. Son autónomas en el plano de la gestión administrativa y pedagógica, pero están fuertemente reguladas por la legislación tanto en la gestión y la rendición de cuentas como en cuanto al respeto de ciertas indicaciones y direcciones de los programas o de la pedagogía en las centros educativos.
} 


\section{Marco teórico y metodológico}

Nuestra problemática se articula en torno a dos preguntas: 1) ¿en qué medida la nueva gestión de la pedagogía engendra nuevas expectativas institucionales por parte de la dirección de los centros de secundaria, que perjudican la autonomía profesional del profesorado?; 2) ¿cuáles son las respuestas que el profesorado de secundaria desarrolla frente a estas expectativas, dado el contexto y del centro donde trabaja?

Arraigamos estas preguntas en la sociología de los grupos profesionales y en los trabajos neoinstitucionalistas sobre la puesta en marcha y la construcción del sentido de las políticas educativas por los actores sobre el terreno.

Para alcanzar los objetivos señalados -principalmente mejorar el logro escolar y reducir el abandono- una política como la GBR pone en marcha nuevas reglas formales, pero vehicula también nuevos marcos cognitivos o una normatividad. Dicho de otro modo, busca hacer evolucionar los acuerdos institucionales que contribuyen a regular y coordinar las conductas de los actores y a darles sentido y estabilidad ${ }^{4}$.

La puesta en marcha de esta política pasa también por un trabajo de los actores de nivel intermedio (CE) o local (dirección de centro) que aplican de manera variable las nuevas leyes, pero contribuyen también a dar sentido a esta política y traducen de ésta de manera más o menos fiel la normatividad. Este trabajo de mediación y de traducción depende de contingencias locales, pero también de los ethos de los agentes de mediación (Maroy, 2010; Spillane, 2002; Spillane, Reiser y Gomez, 2006).

Las expectativas institucionales de la dirección de los centros derivadas de ello pueden ser de naturaleza normativa o coercitiva ${ }^{5}$. Además, se inscriben en los marcos cognitivos y los discursos que las justifican y les dan sentido. Estas demandas normativas o expectativas formales pueden afectar los modos de organización del trabajo o la definición de las profesionalidades de los actores, en particular del profesorado.

Estudiaremos estas demandas y expectativas institucionales a partir de los discursos de los equipos de dirección de los centros, preguntándonos en qué medida inducen cambios en la definición de la profesionalidad del profesorado y contribuyen a redefinir su autonomía profesional, considerada tanto bajo el ángulo del poder de decisión como de la autonomía de reflexión, dos dimensiones clave del profesionalismo según Elliot Freidson (2001). La autonomía de decisión es un tema relativo al derecho de afirmar «su expertise, su propio control del espacio de profesionalidad» (Lang, 1999: 37). En la práctica, esta autonomía es permanentemente negociada con los usuarios, los directivos de las organizaciones que los emplean, así como otros profesionales que forman parte de la ecología de su trabajo (Abbott, 1988). Está asociada a una autonomía de reflexión, que reenvía a una capacidad de reflexión sobre las finalidades, el contexto, pero también sobre los saberes y prácticas a aplicar en el trabajo (Champy, 2009; Tardif y Lessard, 2002).

\footnotetext{
4 De este modo, una política tal busca cambiar las instituciones entendidas en sentido amplio como los marcos reglamentarios y jurídicos, pero también los marcos normativos y cognitivos a partir de los cuales los actores tienden a dar sentido y estabilidad a las relaciones sociales (Scott, 1995). Una práctica institucionalizada tiene tendencia a reproducirse, por una parte porque elle puede imponerse a los actores como un obligación jurídica pero también porque se da por adquirida y considerada como legítima por los actores.

5 Según Coburn (2004, p. 218), una expectativa considerada «normativa» trata sobre las ideas y valores que sustentan lo que el profesorado debe hacer, así como sobre los medios más apropiados con el fin de alcanzar estos nuevos objetivos. Una expectativa «coercitiva» (la autora utiliza el término regulative en inglés) implica una orden directa de cambio (enseñar de una manera precisa, priorizar un cierto material escolar, etc.) que es sustentada por el uso de la regla y por posibles sanciones si el/la docente no se pliega a ella.
} 
Las expectativas institucionales de las direcciones de los centros relativas al profesorado serán analizadas según las tres dimensiones constitutivas del discurso pedagógico para Bernstein (1977). Distinguiremos de este modo las expectativas relativas a los contenidos de enseñana (currículo prescrito y enseñado), al trabajo y a las relaciones pedagógicas en la clase y a los modos de evaluación del alumnado.

Para comprender cómo responde el profesorado a estas expectativas y presiones institucionales, nos referiremos a los trabajos de Coburn (2004). Esta autora muestra que, en muchos casos, los mensajes y las expectativas institucionales afectan al núcleo técnico de la escuela (donde se juega la práctica docente) contrariamente a lo que los primeros neoinstitucionalistas habían adelantado (Meyer y Rowan, 1977). De este modo, para Coburn la asociación entre las expectativas institucionales y las actividades del núcleo técnico es solo un tipo de «respuestas», entre otros. Ella propone distinguir cinco tipos de respuestas del profesorado frente a las expectativas institucionales de cambio de sus prácticas pedagógicas (consultar la Tabla I).

\section{Tabla I. Formas de respuestas del profesorado, adaptadas de Coburn (2004)} TIPO DE RESPUESTA DEFINICIÓN

\begin{tabular}{ll}
\hline Rechazo & La demanda institucional es rechazada, sobretodo si no es congruente con las creencias y visiones del o la docente. \\
\hline Disociación/respuesta simbólica & $\begin{array}{l}\text { La práctica de cada profesor no se afectada en realidad. Se trata más bien de una conformidad simbólica, de una «fachada externa» que no se corresponde con la } \\
\text { práctica efectiva. }\end{array}$ \\
\hline Estructuras paralelas & Frente a las demandas múltiples y contradictorias, el/la docente intenta responder yuxtaponiendo prácticas correspondientes a diferentes expectativas. \\
\hline Asimilación & $\begin{array}{l}\text { El/la docente interpreta la demanda institucional para ajustarla a sus propias creencias y maneras de hacer. Así, la integra a su práctica sin cambiar sus convicciones y } \\
\text { cogniciones de referencia. }\end{array}$ \\
\hline Acomodación & $\begin{array}{l}\text { La demanda institucional implica la transformación de las prácticas y concepciones que la fundan. Esto supone una reestructuración profunda de las creencias de cada } \\
\text { docente, de sus marcos cognitivos }\end{array}$ \\
\hline
\end{tabular}

En el plano metodológico, nuestro trabajo reposa sobre el análisis cualitativo de 20 entrevistas de docentes y 8 miembros de dirección ${ }^{6}$ en el seno de cuatro centros de secundaria en dos CE. El profesorado entrevistado fue seleccionado primero por su antigüedad en el centro (más de 3 años en el caso de 18 de ellos) y su experiencia en el oficio (un promedio de más de 15 años de experiencia). Finalmente, todos enseñaban las materias obligatorias para la consecución de los estudios (la obtención de un diploma al final del programa escolar). Utilizamos las entrevistas de cinco docentes por cada centro estudiado. Primero, hemos realizado la encuesta en tres centros de secundaria de una misma comisión escolar (CE del Norte) que ha sido identificada como «amplificadora» de la GBR y de la gestión de la pedagogía que se sigue de ella (Maroy et al., 2017). Los centros de la CE del Norte han sido seleccionadas en función de la diversidad de sus estudiantes (más o menos vulnerables, provenientes de zonas suburbanas o rurales). Les hemos otorgado los siguientes nombres ficticios: Centro de las Montañas, Centro de las Cataratas y Centro de los Suburbios. En cada uno de estos centros, hemos entrevistado al director del centro así como a uno o dos subdirectores. Con la finalidad de contrastar nuestros datos, hemos continuado la encuesta en otra comisión escolar (la CE del Sur) en la que la lógica de puesta en marcha de la GBR conduce a una gestión de las prácticas pedagógicas locales menos intensa. Por razones ajenas a nuestra voluntad, nuestro plan de investigación (combinando entrevistas a la dirección y al profesorado) pudo ser operacionalizado en un

$6 \quad$ Entre directores y subdirectores. 
solo centro considerado «eficaz o performativo», que llamamos aquí Centro de los Campos. Realizamos en ella las entrevistas a cinco docentes y una sola entrevista a la dirección?

\section{Las expectativas institucionales de los equipos de dirección hacia el profesorado}

Para las direcciones de los centros, la GBR obliga a la reflexividad por parte del profesorado. El análisis de los resultados, y la definición de los objetivos, debe transmitirse al profesorado para promover el principio de reflexión sobre sus prácticas en relación a la preocupación sobre la eficacia pedagógica. Esta expectativa se manifiesta, en todos las direcciones, a través de un discurso donde: 1) el logro académico debe ser la prioridad del profesorado (y del conjunto de los actores); 2) el profesorado debe «cuestionarse»y, en el caso pertinente, replantearse sus formas de hacer en una lógica de mejora continua. Este enfoque conduce a supervisar la autonomía de reflexión del profesorado solo en relación al factor de la eficacia de sus prácticas, y constituye el fundamento de diversas demandas que tienden a reducir su autonomía de decisión.

\subsection{Una obligación de reflexividad instrumental}

En todas los centros estudiados, pero en grados variables, el profesorados debe participar en momentos de reflexión individual o colectiva de sus prácticas. Esta obligación de reflexividad se inscribe en una racionalidad instrumental. La reflexión se focaliza en la eficacia de ciertas prácticas, pero no es suscitada frente a aspectos más amplios, por ejemplo sobre los «valores» o las finalidades educativas del centro, sobre la pertinencia de las herramientas o la finalidad de las políticas. Estos momentos acontecen durante los encuentros colectivos por departamentos (equipos-disciplina, por nivel o interniveles), durante las formaciones dadas por los consejeros pedagógicos ${ }^{8}$, pero también durante las «supervisiones pedagógicas individuales», que puede concernir más raramente al profesorado cuyas prácticas son juzgadas como problemáticas.

Los centros de las Montanas, las Cataratas y los Suburbios forman parte de la CE del Norte, voluntaria en el plano de la gestión de la pedagogía. Los momentos de reflexión colectiva entre docentes son frecuentes y, sobretodo, implican directamente a los equipos de dirección y a los consejeros pedagógicos. Los centros de las Cataratas y de los Suburbios apuestan especialmente por este modelo, particularmente al asociar la reflexividad colectiva con un procedimiento de análisis estadístico de los resultados bastante refinado, y poner el acento en la necesidad del equipo de dirección de comprometerse con el seguimiento y la «regulación» de la pedagogía y del trabajo del profesorado. La dirección del centro de las Montañas es más prudente, porque el profesorado se oponen fuertemente a la intervención de la experticia externa de los consejeros pedagógicos.

Por el contrario, en el Centro de los Campos - dependiente de la CE del Sur- el ensamblaje entre los objetivos que debe alcanzar y las demandas formales de reflexión colectiva hacia el profesorado es más libre. La dirección comunica los objetivos a alcanzar, transmite las expectativas formales sobre los encuentros del profesorado en torno a sus prácticas, pero no sigue ella misma estos encuen-

\footnotetext{
Las referencias a las entrevistas son hechas de la siguiente manera: nombre del centro, función ocupada (con un número si varios entrevistados ocupan una misma función). El profesorado es designado simplemente por su materia. Un director/a de un centro es designado por el acrónimo DE y un director/a adjunto por DA.

8 Los consejeros pedagógicos (CP) tienen, en primera instancia, una función de apoyo a la enseñanza (que puede a veces ser extendida a los equipo de dirección de los establecimientos). Ellos no tienen relación jerárquica con el profesorado. Su rol es entregar una ayuda individual o colectiva en lo que respecta a las metodologías pedagógicas, a la evaluación y al programa de aprendizajes y al material didáctico. Ellos actúan recurrentemente como promotores de la innovación y de las «buenas prácticas», haciendo el puente entre el medio de la investigación y los prácticos. En la CE del Norte, los consejeros pedagógicos dependen jerárquicamente de la dirección del centro y de los servicios educativos de la CE. En la CE del Sur, dependen solamente de los servicios educativos de la CE, pero la dirección del centro puede hacer una petición directa a éstos últimos para tener un apoyo de los CP.
} 
tros. Un «profesor-intermediario» es llamado a establecer la relación entre los «equipos docentes por disciplina»y las demandas de la dirección. La autonomía de reflexión del profesorado se encuentra relativamente preservada y menos controlada, y el uso de los datos es variable.

Este trabajo de reflexividad colectiva se apoya en gran parte en el uso de herramientas estadísticas y en una estrategia central en todos los centros investigadas: hacer visibles ciertos problemas de eficacia pedagógica vía comparación de los resultados. La precisión y la escala de comparación varían sensiblemente entre un centro y otro: comparación con respecto al promedio de la $\mathrm{CE}$, de las materias entre cada centro de la CE, o de diferentes docentes del centro durante un mismo año escolar. Este procedimiento implica una forma de accountability particular, colectiva o individual. El profesorado debe hacer mínimamente inteligibles y justificables sus prácticas a ojos de terceros: sus compañeros, la dirección, incluso las familias (consultar Ranson, 2003). No se trata de una forma de «accountability dura» acompañada de amenazas para su empleo, sus salarios, o sus ventajas (Maroy y Voisin, 2014). Se trata fundamentalmente de una demanda de reflexividad sobre su eficacia, que pone a prueba sus competencias profesionales, cuando sus prácticas parecen fuera del estándar de resultados, o fuera del campo de lo que es prescrito o legítimo.

En la CE del Norte, el director del Centro de las Montañas destaca de este modo la necesidad de confrontar las «cifras», comparando diferentes resultados, con la finalidad de que el profesorado «progrese».

"Los reciben mejor que antes, porque traté de formarles sobre ello, yo decia "no os sintáis amenazados, yo no estoy aqui para echaros, no estamos aqui para juggar si eres bueno o no". Lo que yo quiero es que las personas caminen, que progresen». (Centro de las Montañas, DE).

La demanda de justificaciones toma también la forma de un cuestionamiento o de un diálogo colectivo sobre las prácticas docentes. Un director adjunto administra por ejemplo la reflexión, lo que le permite «ver» las lagunas potenciales del profesorado en relación con las prescripciones curriculares:

"Aqui [en las discusiones colectivas], no pueden esconderse detrás de los compañeros, o detrás de la puerta cerrada de su clase. Aquí, las discusiones, lo que se escucha, lo que se ve, nos lleva a hacer constataciones también. Tú sabes: ¿quién posee sus cosas? ¿Quién patina un poco en sus cosas? Aquí lo vemos concretamente. Entonces, eso, es interesante». (Centro de las Cataratas, DA).

Por otro lado, en todos estos centros, los equipos de dirección ejercen un seguimiento individual de los resultados. Este seguimiento puede conllevar una solicitud de justificación a un/a docente. Ese/a docente en cuestión suele tener dificultades para alcanzar los objetivos fijados, o sus clases presentan resultados marcadamente diferentes con respecto a sus compañeros: «Si es frecuente que los resultados de un profesor sean siempre bajos, voy a tratarlo individualmente [... J》. (Centro de las Montañas, DA).

Cada docente debe dar una explicación válida a un problema identificado y comprometerse a modificar sus prácticas con la finalidad de corregirlo. Esta explicación podrá, en el caso pertinente, ser transmitida a las familias.

«Elprofesor es seguido casi mes a mes por la dirección adjunta responsable de su supervisión: se ven sus exámenes, sus resultados. Antes del encuentro del informe de notas, vamos a entregar sus resultados porque sabemos que hay muchos padres que van a ir a verlas». (Centro de los Suburbios, DE). 
Finalmente, los intentos del profesorado de culpar a los factores contextuales que los liberarían de «sus responsabilidades» son desechados. Los efectos contextuales son tomados en cuenta, pero no constituyen un argumento válido para ignorar el efecto del o la docente.

"[...] hay factores personales [de los alumnos] que pueden jugar, hay factores de la cohorte. Hay toda una gama de factores, tú sabes, es necesario tenerlos en cuenta [...] no habria que [centrarse] únicamente en los resultados [...] [Pero] un poco en paralelo a ello, yo no soy del tipo que acepta las excusas de un profesor que va a esconderse detrás de los múltiples [factores externos], tú sabes». (Centro de las Cataratas, DA).

\subsection{Los límites de la autonomía de decisión del profesorado}

La voluntad de las direcciones de limitar la autonomía de decisión del profesorado emerge claramente de nuestros análisis, particularmente en lo que se refiere a las prácticas de evaluación del alumnado y a la cobertura de los programas. La autonomía de decisión del profesorado en materia de práctica pedagógica en clase sigue siendo importante ${ }^{9}$. Las demandas de cambio son también más numerosas y más precisas en los centros de la CE del Norte con respecto al Centro de los Campos.

Las demandas vinculadas a las prácticas de evaluación

Las direcciones consideran que las prácticas de evaluación deben ser controladas, ya que éstas tienen un vínculo directo con los resultados del alumnado, su perseverancia y la obtención del diploma. Los exámenes ministeriales, obligatorios para la obtención del diploma, deben transformarse en un patrón externo en relación al cual ajustar las prácticas de evaluación en las clases. En todos los centros, el profesorado es alentado a concentrar sus evaluaciones sobre los elementos que serán preguntados en los exámenes ministeriales. Además, las prácticas evaluadoras no deben divergir de las normas ministeriales de evaluación. Por ejemplo, un director adjunto en el Centro de las Montañas pide al profesorado que deje de evaluar la ortografía fuera de las pruebas específicamente reservadas a la escritura en nombre de las «indicaciones ministeriales» a seguir.

Asimismo, las prácticas correctivas del profesorado deben seguir también los estándares ministeriales. Cada docente debe evitar que se cree una distancia entre las notas obtenidas por sus estudiantes durante el curso y aquellas obtenidas en los exámenes ministeriales. Una buena corrección debe ser «realista», por lo tanto llevar a una nota que no será objeto de un ajuste ministerial al final del año.

Finalmente, hay una presión ejercida sobre el profesorado con la finalidad de que armonice sus formatos y prácticas de evaluaciones a través de una autorregulación del colectivo docente y a través del recurso a una formación sobre los criterios de evaluación de las pruebas ministeriales. En el Centro de los Campos, se le pide al profesorado introducir pruebas comunes finales (hechas por los equipos disciplinares) en los niveles y materias donde no existen exámenes ministeriales.

\section{Las demandas vinculadas al contenido enseñado}

Las demandas de conformidad al programa (currículo nacional) están asociadas al GBR en la medida en que ello permite preparar mejor al alumnado para las pruebas externas y entregar puntos de comparación más sólidos. Las elecciones didácticas del profesorado deben hacerse en los límites del currículo prescrito por el ministerio, precisado en la herramienta llamada Progresión de los Aprendizajes (PDA), es decir un complemento al programa que detalla, cada año, para cada materia, los conocimientos que el alumnado debe adquirir.

9 Sin embargo, puede indirectamente ser afectada por la obligación de la reflexividad; sin responder a una orden formal, el profesor cambia sus prácticas, tras evidenciar un mal rendimiento. 
En los Centros de las Montañas, las Cataratas y los Suburbios, hay una tendencia de conformidad fuerte de cada docente a la PDA. El respeto a la PDA apunta a evitar que ciertos aspectos del programa sean ignorados o tratados insuficientemente en un momento u otro del curso escolar de cada estudiante, y esto incluso si tales aprendizajes están sugeridos en los libros de texto o son considerados importantes por el profesorado.

Además, el profesorado es llamado a trabajar colectivamente con el fin de asegurar que la progresión didáctica sea respetada. El rol de las direcciones adjuntas consiste en asegurar la puesta en marcha de este trabajo colectivo:

«El seguimiento colectivo lo hago verdaderamente en el marco de aquello [que] les he comentado hace un rato. Yo parto verdaderamente de la Progresión de los Aprendizajes [...] para asegurarme de que cada parte de la materia sea realmente vista en el nivel que corresponde, donde eso debe ser visto» (Centro de las Cataratas, DA).

Para las direcciones, la PDA debe favorecer la comunicación entre el profesorado con la finalidad de su autorregulación desde la perspectiva de los contenidos curriculares prescritos.

Por otro lado, las evaluaciones ministeriales apuntan explícitamente a una parte de los contenidos precisados en la PDA, para cada año de estudios concernido. El acento puesto sobre los resultados del alumnado en las evaluaciones ministeriales crea un imperativo didáctico para el profesorado. De este modo, las direcciones hacen demandas de abandono de prácticas de enseñanza sobre objetos que no son considerados «necesarios», ya que no están previstos en la evaluación externa del alumnado.

Las demandas vinculadas a las prácticas pedagógicas en clase

En las teorías de la acción asociadas a las políticas de accountability, las prácticas pedagógicas son centrales como objeto de cambio implicado (Fullan, 2007). En la práctica, estas expectativas de cambio de las prácticas pedagógicas están presentes en las convenciones de gestión firmadas entre los centros y las CE, ya que los «medios» de estas convenciones, destinados a mejorar los resultados del centro, implican a menudo las prácticas en clase. Paradójicamente, las demandas y expectativas de las direcciones son, en este tema, menos directas o explícitas y conllevan fundamentalmente una llamada general al cambio (sobre todo a través de la reflexión sobre los resultados).

En raros casos, las prácticas pedagógicas son objeto de demandas explícitas y formales por parte de las direcciones ${ }^{10}$. Se trata, por ejemplo, de transmitir un modelo pedagógico promovido por la $\mathrm{CE}$, destinado a mejorar los resultados en lectura. Es el caso de todos los Centros de la CE del Norte, el centro de las Montañas y el de las Cataratas, que toman esa estrategia como «medio» para alcanzar un objetivo de su convención de gestión.

"[Este modelo] es a pesar de todo una orientación de la comisión escolar, indirectamente se evalúa la lectura en secundaria II. Se sabe que con [este modelo] somos capaces... tenemos buenos resultados. Con este medio indirecto, somos capaces de cambiar prácticas [del profesorado en clase]». (Centro de los Suburbios, DE).

10 Aunque la libertad de elección pedagógicas del profesorado esté inscrita en la Ley sobre la instrucción pública (art. 19), la CE puede hacer una demanda de cambio de ciertas prácticas pedagógicas docentes a la dirección de los centros. 
No obstante, las demandas asociadas a las prácticas pedagógicas son mayoritariamente indirectas. La demanda de reflexión sobre las prácticas docentes implica, explícita o implícitamente, una demanda de cambio de estas prácticas cuando los resultados no son alcanzados. A menudo, esto equivale a aplicar las sugerencias de los consejeros pedagógicos o a emular las prácticas de sus pares (tomando como recurso a docentes-referentes) que parecen obtener resultados en el plano de los resultados académicos, de la perseverancia escolar o de la gestión de clase.

\section{La diversidad de las respuestas docentes}

Hemos identificado entre el profesorado de nuestra muestra tres grandes tipos de respuesta, elaboradas reagrupando las categorías de Coburn. El primer tipo de respuesta procede de una aceptación relativa de la demanda institucional: el profesorado responde a las órdenes articulándolas en su práctica existente, sin cambiar sus convicciones y cogniciones de referencia. El segundo tipo de respuesta (el más presente en nuestro corpus) es la oposición relativa a las demandas institucionales que dan lugar a formas de rechazo explícito y, en menor medida, a formas de respuestas simbólicas o de estructuras paralelas. Finalmente, hemos identificado un tercer tipo de respuesta especifica a nuestro corpus que calificamos de «renuncia», es decir una forma de conformidad obligada que no muestra ni aceptación ni oposición. La Tabla II presenta la distribución relativa de las codificaciones efectuadas en el conjunto de nuestro corpus.

Tabla II. Repartición de la codificación según las categorizaciones de las respuestas

\begin{tabular}{lllll} 
TIPOS DE RESPUESTA & FRECUENCIA & TIPO DE RESPUESTA & FRECUENCIA & $\%$ \\
\hline 1: Acomodación & 8 & Aceptación relativa & 79 & $41 \%$ \\
\hline 2: Asimilación & 71 & & & \\
\hline 3: Disociación/Respuesta simbólica & 18 & & 42 & $42,5 \%$ \\
\hline 4: Rechazo & 55 & Oposición relativa & & $16,5 \%$ \\
\hline 5: Estructuras paralelas & 9 & & 32 & \\
\hline 6: Renuncia & 32 & Renuncia & & \\
\hline
\end{tabular}

Las subsecciones siguientes abordan sucesivamente cada uno de estos tres tipos de respuestas, en relación a la dimensión de la actividad pedagógica (contenidos enseñados, trabajo y relaciones pedagógicas en la clase, evaluación) a las que se refieren con más frecuencia.

\section{1. La aceptación relativa: una congruencia parcial entre las demandas institucio- nales y las creencias y prácticas del profesorado}

Hemos reagrupado en un primer tipo de respuestas aquellas que manifestaban una forma de recepción positiva o, al menos, de aceptación relativa de las demandas institucionales. Este tipo de respuesta está asociado a las demandas sobre las secuencias de contenidos docentes, a las demandas de ajuste de las prácticas de evaluación con respecto a las expectativas del ministerio, y a una demanda de reflexividad sobre los resultados, tres aspectos ligados a mejores resultados del alumnado.

En lo referente, en primer lugar, al dominio de los contenidos del programa que debe ser enseñado, se observa una demanda del profesorado por referencias precisas en cuanto a la articulación del programa a sus prácticas: «es vital en francés saber cuál es la materia que debo cubrir en el año» (Centro de las Cataratas, Francés n. 2). La demanda de la dirección de aplicar la PDA se une bastante bien a 
sus expectativas; es asimilada a sus prácticas. El profesorado ven en ella, entre otros elementos, una manera de aumentar los resultados de sus estudiantes, ya que enseña más o menos lo que será exigido en el examen del ministerio.

El profesorado dice «demostrar criterio» (Centro de las Montañas, Francés n. 2) para identificar las prioridades en términos de contenido de enseñanza, siendo siempre su objetivo final el logro de sus estudiantes. Algunos insisten en que llevan la iniciativa de sus elecciones: «la demanda, venía del profesorado» (Centro de las Cataratas, Matemáticas). Se acepta la demanda de autorregulación colectiva dirigida por la dirección al profesorado en lo relativo a las secuencias de una materia según la PDA. De hecho, algunos docentes apelan a una responsabilidad del colectivo docente y justifican las elecciones comunes de cobertura del programa:

"[...] Si [nuestro equipo de francés] es verdaderamente eficaz, en esto, es que está verdaderamente bien concebido, en todo caso comunicamos bien de un nivel a otro, una vez. llegados a quinto de secundaria, si todo el mundo trabaja en la misma dirección, deberíamos ser formidables». (Centro de las Cataratas, Francés n. 2).

En materia de evaluación, las respuestas de tipo asimilación se manifiestan sobre todo con respecto a las demandas de armonización de las prácticas evaluativas. El profesorado no se cuestiona la preponderancia de los patrones que son, de facto, los exámenes del Ministerio y su importancia en el logro del alumnado. Hay aquí una aceptación de una limitación de la autonomía de decisión relativa al nivel de logro del alumnado en los exámenes. Estas demandas parecen relativamente bien institucionalizadas y aceptadas: «[...] se cambian los modos de evaluación para adaptarse al modo de evaluación del ministerio» (Centro de las Cataratas, Matemáticas).

En el mismo espíritu, algunos docentes han tenido iniciativas con el fin de hacer corresponder la ponderación de sus evaluaciones en clase a aquella presente en los exámenes de ministerio. Las expectativas institucionales se incorporan, en este caso, a las concepciones y prácticas existentes, sin modificarlas fundamentalmente.

"[...] Lo que se ha intentado es, [por un lado], trabajar sobre la ortografía, el uso gramatical. Es el criterio más fallado en el examen, y, [por otro lado], cambiar de ponderación en nuestro examen de gramática. [...] Es mi iniciativa en este caso, porque quiero que mis alumnos triunfen [...] Por otro lado, hago mucho énfasis en la prueba del Ministerio, enormemente». (Centro de las Montañas, Francés n. ${ }^{\circ}$ 1).

Finalmente, con respecto a la relación del profesorado con la obligación de reflexividad, se observa en la mayoría de los casos una fuerte congruencia entre las expectativas normativas de la dirección de los centros y la manera en la que el profesorado ve su propia identidad profesional: «es normal que yo me cuestione sobre el logro de mis estudiantes» (Centro de los Suburbios, Francés, n. ${ }^{\circ}$ 2). El profesorado comparte la preocupación del éxito de todo el alumnado (al menos el suyo) y convierte en cuestión de honor hacer sus prácticas más eficaces.

"[...] tú sabes, quiero decir yo, todos mis resultados desde que enseño, yo quiero que mis alumnos triunfen, está claro, me evalúo yo mismo, con respecto a eso, es seguro, pero es seguro que eso [hablando de la evaluación externa] puede crear problemas». (Centro de los Suburbios, Matemáticas n. ${ }^{\circ}$ 2). 
En el Centro de las Cataratas, un profesor explica incluso haber cambiado de posición con respecto a la GBR y valora el cuestionamiento sobre los resultados de sus estudiantes que la misma conlleva. Se trata aquí de una forma de «acomodación» de la demanda institucional, es decir, que la integración de la demanda va acompañada de una transformación de las creencias, de las estructuras cognitivas del docente.

«Sin embargo, al principio yo era una de las personas que [decian]: [...] 'ellos van a darnos un bono por el rendimiento de los alumnos?' No tienen ningún sentido en educación estas cosas. Al principio, yo estaba en esta óptica, ahora, cuanto más pasan los años, más lo veo: sí, puede ser útil. [...] Cuando nos asentamos, cuando buscamos los medios, yo pienso que se puede estar más concienciado. Cuando se es capaz de encontrar maneras de hacer en la escuela que pueden ayudar a estos jóvenes. Porque yo pienso que se han puesto los recursos para ello, yo creo que marcha, que eso funciona». (Centro de las Cataratas, Matemáticas).

La congruencia y la adhesión cognitivas y normativas a las expectativas de la dirección están, sin embargo, lejos de ser totales. Así, en el plano cognitivo, el ideal de «logro para todos» (y de perseverancia de todos) fuertemente promovido por la dirección es, a veces, adoptado de manera ambigua por el profesorado. El ideal de una igualdad de oportunidades meritocrática y la idea de que es necesario crear condiciones favorables al logro de todos (prestando a veces mayor atención a las personas más débiles, por ejemplo) están efectivamente presentes. No obstante, esto no significa para el profesorado que todos el alumnado va a tener éxito o lograr el mínimo de conocimientos esperados -la nota para aprobar el examen ministerial-. El logro depende también del esfuerzo y de las aptitudes (variables) de éstos últimos. El argumento puede resumirse de la manera siguiente: el profesorado tiene un trabajo que hacer (implantar medidas para favorecer el logro), pero el alumno tiene también que realizar un trabajo (realizar los «esfuerzos» y utilizar estas medidas). Dicho de otro modo, la aspiración y las condiciones de logro de todos pueden a veces ser entendidas de manera diferente según los actores escolares que pueden asociarlas a referentes normativos distintos en materia de justicia escolar (igualdad de conocimientos vs igualdad de oportunidades, consultar Crahay, 2012).

Observamos, en este sentido, una congruencia parcial entre la visión del profesorado y aquella de las direcciones. Sin embargo, numerosos desacuerdos, desfases o malentendidos cognitivos se manifiestan más allá de este acuerdo parcial. Se observan también numerosas prácticas cuyo sentido no es compartido entre el profesorado y la dirección del centro. Abordamos esta cuestión en el siguiente subapartado.

\subsection{Oposiciones a las demandas normativas de la dirección de los centros: recha- zos y respuestas simbólicas}

Están igualmente presentes en nuestro corpus algunas formas de oposición más o menos explícitas, relativas principalmente a las demandas sobre las prácticas pedagógicas así como las demandas vinculadas al «nivel de exigencia» de las prácticas evaluadoras. En lo que respecta a la evaluación, el profesorado revindica a menudo un nivel de exigencia (en la corrección) más severo que lo que está recomendado por el mensaje institucional. Algunos hablan de «resistencia», como este profesor de matemáticas: «Todo lo que me queda es el rigor al nivel de la corrección» (Centro de las Montañas, Matemáticas n. ${ }^{\circ}$ 1). En este caso, el profesorado apela al logro de sus estudiantes para justificar el mantenimiento de estas prácticas de corrección. 
«No estoy listo para decir, 'bueno ok con eso voy a dejar pasar ciertas cosas, voy a ser menos exigente para aumentar mis resultados', si yo insisto en que tengan éxito en estas cosas, es porque quiero que sean capaces de hacerlo». (Centro de las Montañas, Matemáticas n. ${ }^{\circ}$ 2)

La demanda institucional es entonces rechazada o fuertemente desacoplada de la práctica efectiva. Así, numerosos docentes, sin cuestionar el principio de ajuste de sus notas al ministerio, rechazan las demandas relativas a una evaluación «realista» durante el año, porque inciden en sus estrategias de movilización del alumnado. La dirección de los centros quisiera alinear sus prácticas de evaluación con el patrón de las pruebas externas (del ministerio o de la $\mathrm{CE}$ ), las cuales les reprochan su «severidad» (con el riesgo de desmotivar y de aumentar el abandono, sin contar con que haga caer el promedio de los resultados del centro, basados en las notas obtenidas en el transcurso del año y no solo en la prueba externa). Por contra, el profesorado revindica esta severidad, en nombre precisamente de una estrategia de «logro» basada en una concepción relativamente utilitarista del alumnado (sensibles a su calificación) y en una teoría práctica de lo que afecta o favorece su motivación. De ahí la resistencia que justifican en nombre de la mejora y del logro efectivo de sus estudiantes.

«Porque los resultados no son siempre buenos durante el año. Esto provoca que [la dirección] tema que los alumnos se desmotiven, para luego abandonar el curso. Pero dado que soy consciente de que corrijo más severamente, según les explico, les recuerdo regularmente que el objetivo es que, si encuentras eso difícil, pero lo logras, cuando llegues al examen del Ministerio, deberías encontrarlo mucho más fácills. (Centro de las Montañas, Matemáticas n. ${ }^{\circ}$ ).

Notamos igualmente que en casos más raros, el profesorado declara abiertamente echar mano a prácticas que podemos caracterizar como respuestas simbólicas, y que apuntan a disociar la demanda institucional y el núcleo de su práctica efectiva.

«Eso está claro, que la presión es muy, muy fuerte aquí. Yo, yo formo parte de los últimos aquí, quien va a paso de tortuga. Me apuntan con el dedo porque... corregir con patrones... yo, yo voy a HACERLO, pero yo adapto el patrón a mi manera de corregir (risas) por eso... yo hago un poco trampa! [...] $Y$ mi resultado va a ser seguramente más severo que el del otro profe que corregiría el mismo examen que yo porque... es así como yo veo las cosas». (Centro de las Montañas, Matemáticas n. ${ }^{\circ}$ ).

Vemos aquí un mal entendido entre dirección y profesorado (sobre lo que es una gestión eficaz del «logro» del alumnado), más allá de un acuerdo superficial sobre el objetivo de éxito. Este malentendido conduce a una divergencia -entre dirección y profesorado- sobre lo que es considerado como el referente pertinente en la óptica del «éxito». Por una parte, la dirección de los centros ve el logro de «todo el alumnado» a través de ciertas estadísticas preponderantes, es decir, de los promedios de éxito en una prueba o las tasas de diplomados del centro. Por otra parte, el profesorado da prioridad a la preocupación por su alumnado y contempla la estrategia de «contribuir al éxito» en relación a personas concretas. Los resultados estadísticos de todo el centro serán siempre secundarios con respecto a su objeto de preocupación más inmediato: «mi clase», «mis alumnos». Es también la consideración del contexto particular (de la composición de la cohorte de estudiantes, las particularidades de los alumnos) lo que conduce a numerosos docentes a tomar distancia con respecto a una reflexividad colectiva sobre los resultados cuantificados, sobre todo cuando ésta supone una comparación.

Con respecto a las prácticas pedagógicas en el seno de la clase, el margen de maniobra del profesorado parece permitir fundamentalmente el rechazo explícito. En el Centro de las Montañas, el profesorado 
expresa su rechazo absoluto a prácticas tales como el trabajo en equipo y la pedagogía del proyecto, y revindica una enseñanza magistral, prácticas donde cada estudiante se entrena solo, preparándolo de este modo fundamentalmente para los exámenes del ministerio: «Yo, los conceptos, los presento una vez. Luego el resto es magistral...» (Centro de las Montañas, Historia).

Así, ciertas teorías pedagógicas promovidas o prescritas por la CE y la dirección son, a veces, puramente y simplemente rechazadas. Esta no-congruencia sobre las creencias pedagógicas puede explicar las tensiones con los consejeros pedagógicos.

«Mi margen de maniobra está en la manera en la cual yo realizo mi enseñanza. Yo enseño mi programa como lo deseo, como yo lo quiero y, por el momento, me abstraigo de las sugerencias que han podido serme hechas por el consejero pedagógico». (Centro de los Campos, Historia).

La oposición a las demandas de la dirección de los centros puede igualmente traducirse a través de la formas de estructuras paralelas, donde el docente yuxtapone diferentes enfoques pedagógicos para responder a las demandas en tensión. Algunos docentes declaran así consagrar una parte del tiempo en clase al modelo de enseñanza eficaz en lectura, conservando al mismo tiempo sus prácticas anteriores para otros momentos, estableciendo así una suerte de equilibrio entre diferentes prioridades.

\subsection{La renuncia o la conformidad obligada: cuando lo «deseable» deviene una práctica impedida}

Un tercer tipo de respuesta ha emergido de nuestro corpus. Se trata de respuestas donde el profesorado «renuncia» a una práctica que juzga deseable y aplica una práctica conforme a la demanda institucional sin modificar por ello sus concepciones, sus valores. Esta conformidad resulta un obstáculo, formas de regulación que imponen una acción incluso si ésta es juzgada ilegítima. En efecto, la renuncia concierne sobre todo a prácticas sobre las que pesan a la vez la posibilidad de un control ex post a través de los resultados (por lo tanto la posibilidad de visibilización), la demanda de respeto de una herramienta prescriptiva (por ejemplo la PDA) y, finalmente, una fuerte preocupación organizacional (el imperativo de rendimiento). La renuncia no puede ser asimilada a otras respuestas distinguidas por Coburn. No es ni un rechazo ni una forma de disociación ya que la práctica del profesorado está efectivamente adaptada en función de la expectativa institucional. Tampoco se trata de un caso de estructuras paralelas, porque esta práctica adoptada por el profesorado no cohabita con otra opuesta. Finalmente, no es tampoco una adopción de una práctica que se alía completamente con un cambio profundo de las creencias (acomodación) o que sea compatible con los valores y cogniciones previas del profesorado (asimilación) porque el o la docente actúa a su pesar.

Observamos este tipo de respuesta en todos los centros de nuestra muestra con respecto a los contenidos que se deben enseñar y, más marginalmente, con respecto a ciertas prácticas de evaluación, es decir, en dominios donde hemos identificado una fuerte pérdida de autonomía de decisión.

Las presiones institucionales que conciernen los contenidos de enseñanza forman parte de las prácticas cuyo sentido no es siempre compartido entre el profesorado y la dirección de los centros. Cierto profesorado expresa efectivamente dudas en cuanto a los límites de los aprendizajes preconizados a través del «respeto estricto de los programas». Aún reconociéndole ciertos efectos positivos, cuestiona los efectos de la PDA ya sea con respecto a un objetivo más general de la materia, o bien con respecto a la relación pedagógica: 
«Bueno, a corto plazo, esto funciona y es maravilloso. Ése es el objetivo, que ellos obtengan su diploma de estudios secundarios, pero tú te dices, ¿es el mejor método? [...] ¿V Van a salir siendo ciudadanos tan completos como si no bubiéramos respetado eso? Es aqui que, a veces, tienes que matizar, sí, no estoy tan seguro». (Centro de las Cataratas, Historia).

El profesorado renuncia a una práctica juzgada deseable para conformarse con la demanda institucional: «Me gustaba ir más lejos con los alumnos [...] Pero abora, bien, es tan ajustado, tan ajustado que, en un momento dado, se corta con ello» (Centro de las Montañas, Matemáticas n. ${ }^{\circ} 1$ ). Sea cual sea la materia, el programa es más ajustado durante un año de evaluación. Así, este profesor de historia (Centro de los Campos) dice estar fuertemente contrariado en sus elecciones de enseñanza por la exigencias del examen ministerial: «Estoy completamente limitado, la presión es enorme. [...] Yo pongo énfasis en las probables preguntas del examen».

En este contexto, el profesorado acepta abandonar ciertos contenidos, pero a su pesar, consciente de que su docencia deviene más delimitada por exigencias externas. Por ejemplo, otro profesor de historia lamenta el abandono de las salidas educativas. Asimismo, una profesora de francés en último año de secundaria (año del examen) explica lamentar ver la poesía solo «al vuelo», ya que, aunque está presente en el programa, no es objeto de evaluación ministerial. Así, numerosos profesores ajustan sus prácticas docentes en función de las evaluaciones externas obligatorias, lo que corresponde a lo que las investigaciones norteamericanas han calificado de teaching to the test (Rozenwajn y Dumay, 2014), pero algunos lo hacen con un sentimiento de lamento, cuestionando la pertinencia de sus elecciones.

Si otros profesores afirman poner énfasis sobre los contenidos evaluados por iniciativa propia, para que «[sus] alumnos lo logren», aquí la práctica es más ambivalente. La renuncia puede estar ligada a una forma de cuestionamiento y de autorregulación donde el profesorado establece pragmáticamente la jerarquía de lo que está en juego: es importante ver la poesía con el fin de favorecer el desarrollo del alumnado, pero es todavía más importante que el alumnado apruebe sus exámenes y pueda pasar al nivel superior. El profesorado ajusta entonces sus prácticas con coherencia a esto, pero esta jerarquía de los elementos en juego crea una tensión identitaria que pone a prueba la visión de las finalidades del oficio:

«Si, es bueno que pasen el examen del ministerio, pero a menudo es en detrimento de la literatura, en detrimento de otra cosa, por eso hasta qué punto enseñamos para la evaluación, hasta qué punto enseñamos el resto de la nociones». (Centro de los Suburbios, Francés n. 1).

En lo que respecta a ciertas prácticas evaluadoras, esta respuesta aparece más específicamente ligada al profesorado del Centro de las Montañas. Así, la orden de la dirección que les obliga a abandonar la evaluación de la calidad de la lengua en todas las materias es interpretada como una valoración artificial de los resultados escolares, sintomática de una estrategia de «aparentar» por parte de la CE, en detrimento del aprendizaje del alumnado ${ }^{11}$. El profesorado están a pesar de todo obligados a conformarse a la demanda, porque estas prácticas son «visibles» y por ello potencialmente objeto de control de los padres y las direcciones.

11 La calidad del lenguaje se refiere a ortografía, gramática, sintaxis. En otras palabras, se pide al profesorado (matemáticas, historia, ciencia...) que ignoren estos aspectos, que se refieren a la calidad del lenguaje y a su dominio por parte del alumnado en sus evaluaciones. Sin embargo, el profesorado que imparte lengua francesa sí sigue evaluando la calidad de la lengua francesa. 


\section{Respuestas contrastadas según los centros}

Hemos mostrado hasta aquí las múltiples significaciones que las demandas formales o las expectativas normativas más difusas de la dirección de los centros investigados pueden suponer para el profesorado, al implementar la GBR. Esta variedad de significaciones se traduce en respuestas docentes que toman dos formas mayores y una forma menor: una aceptación relativa, una oposición relativa y, de manera menos importante, la forma de una renuncia o de una práctica impedida por las presiones institucionales ejercidas por la dirección. Las respuestas del profesorado a las presiones institucionales son variadas, pero también complejas en su significación individual en la medida en que, de manera recurrente, un mismo docente desarrolla numerosos tipos de respuesta en función de las demandas y presiones institucionales a las cuales se enfrenta, y del tipo de práctica concernida.

Sin embargo, si se observan y comparan las respuestas según el centro (Tabla III), se percibe que las respuestas modales o las frecuencias relativas de respuestas varían significativamente entre un centro y otro. Los Centros de las Montañas y de los Campos tienen un fuerte porcentaje de «codificaciones» de respuesta de su profesorado en la categoría de la oposición relativa (respectivamente $60 \%$ y $48 \%$ contra 42,5\% para el conjunto del profesorado) mientras que los Centros de las Cataratas y de los Suburbios tienen como categoría de respuesta más frecuente la aceptación relativa (52\% y 49\% contra $41 \%$ para el conjunto de la muestra).

Tabla III. Frecuencias de los códigos por categorías de respuesta, comparación por centro

\begin{tabular}{|c|c|c|c|c|}
\hline & $\begin{array}{l}\text { ACEPTACIÓN } \\
\text { RELATIVA/INTEGRACIÓN } \\
\text { A LAS PRÁCTICAS }\end{array}$ & $\begin{array}{l}\text { RECHAZO Y RESPUESTA } \\
\text { SIMBÓLICA }\end{array}$ & RENUNCIA & TOTAL \\
\hline \multirow{2}{*}{ Centro de los Campos } & 12 & 15 & 4 & 31 \\
\hline & $39 \%$ & $48 \%$ & $13 \%$ & $100 \%$ \\
\hline \multirow{2}{*}{ Centro de las Montañas } & 15 & 36 & 9 & 60 \\
\hline & $25 \%$ & $60 \%$ & $15 \%$ & $100 \%$ \\
\hline \multirow{2}{*}{ Centro de las Cataratas } & 33 & 22 & 8 & 63 \\
\hline & $52 \%$ & $35 \%$ & $13 \%$ & $100 \%$ \\
\hline \multirow{2}{*}{ Centro de los Suburbios } & 19 & 9 & 11 & 39 \\
\hline & $49 \%$ & $23 \%$ & $28 \%$ & $100 \%$ \\
\hline \multirow{2}{*}{ Total } & 79 & 82 & 32 & 193 \\
\hline & $41 \%$ & $42,5 \%$ & $16,5 \%$ & $100 \%$ \\
\hline
\end{tabular}

Por ello, hay también dinámicas que se generan a nivel de centro. Para desarrollar la inteligibilidad, nos limitamos, en esta discusión, a algunas hipótesis -alimentadas por nuestro estudio empírico-. De este modo, nos parece que la variación de las respuestas del profesorado entre centros podría ser aclarada por, al menos, tres elementos y condiciones en interacción. Estas respuestas son influenciadas, por una parte, por el contexto de los centros educativos (su población estudiantil, el nivel de sus resultados, la historia del centro) y, por otra parte, por las estrategias - más o menos directivas o participativas-adoptadas por su dirección (y, más allá, por la CE) para poner en marcha la GBR, la cual no es independiente de las culturas e identidades del profesorado con el que interactúan. Así, la configuración de las relaciones entre los actores de los centros podría ser bastante decisiva en la interpretación de las diferencias. Este hecho se puede ilustrar a partir de los centros investigados. 
Los Centros de las Montañas y de los Campos presentan un porcentaje importante de docentes que manifiesta formas de oposición relativa a la regulación de las prácticas pedagógicas. Son centros relativamente privilegiados y eficaces en cuanto a los resultados. Por el contrario, las presiones y estrategias de gestión de la pedagogía desde la dirección contrastan claramente y afectan de manera variable el espacio de autonomía de decisión del profesorado, a nivel individual o colectivo.

El Centro de las Montañas es, así, relativamente eficaz en el logro de resultados, pero su dirección busca introducir de manera progresiva una lógica de regulación colectiva de las prácticas de evaluación y un seguimiento más escrupuloso de la PDA, garantía de mejores resultados esperados por la CE del Norte que, recordémoslo, es proclive a la puesta en marcha de la GBR. Tiene la voluntad de intervenir en el espacio de autonomía del profesorado, de manera que se producen tensiones entre el profesorado y la dirección del centro bastante fuertes (sobre todo a nivel sindical), y el profesorado desarrolla, con frecuencia, respuestas de oposición a la GBR. La cultura del profesorado se describe así por numerosos interlocutores como aquella «de irreductibles Galos» opuestos al modernismo pedagógico encarnado fundamentalmente por los consejeros pedagógicos, que raramente se aventuran. La estrategia de la dirección se adapta a estas resistencias, pero las suscita igualmente, porque toca la autonomía de decisión del profesorado.

El Centro de los Campos pertenece a la CE del Sur, menos ofensiva que la CE del Norte en la puesta en marcha de la GBR. La dirección interviene poco en la zona de autonomía profesional del profesorado cuando se trata de aplicar la GBR. Se despliega a través de una estrategia de cooptación de docentesreferentes, que se encargan de pasar el mensaje institucional, por ejemplo, de convencer y movilizar al profesorado en una tendencia hacia una armonización de las prácticas de evaluación. Por otro lado, no manifiesta las expectativas particulares en materia de métodos pedagógicos ni refuerza, a través de su propio discurso institucional, las prescripciones curriculares del ministerio. Esta situación podrían estar vinculada, por una parte, al hecho de que el centro ya tiene buenos resultados y comparte, según ciertos interlocutores, una «cultura de un buen centro» reputado en la región; por otra parte, que la dirección está preocupada, a través de su estrategia participativa, de no herir de frente al profesorado celoso, sobre todo en el plano sindical, de preservar su autonomía. Las oposiciones relativas a la GBR -que podrían parecer paradójicas- juegan, sin duda, un rol expresivo y defensivo que apunta a prevenir toda intrusión de la dirección en su zona de competencia, lo que ha contribuido a la prudencia en la implementación de la GBR en este centro.

Los otros dos Centros de la CE del Norte - de los Suburbios y de las Cataratas-contrastan desde el punto de vista de la población y de los resultados. El de los Suburbios es, inicialmente, un centro con bajos resultados y desfavorecido ${ }^{12}$, mientras que el de las Cataratas es, a la vez, eficaz en cuanto a los resultados y privilegiado. Por el contrario, en ambos centros las frecuencias relativas de aceptación de la GBR son las más importantes (sobre la media de la muestra codificada) por parte de los equipos docentes. Sin embargo, esta situación va de la par, por un lado, con una estrategia ofensiva de la dirección en materia de gestión de la pedagogía, al mismo tiempo que con la capacidad de la dirección de instaurar en los dos casos formas de colaboración y de participación entre profesorado y dirección del centro.

El Centro de los Suburbios depende de la CE del Norte, que espera mejoras sustanciales de los resultados. Simultáneamente, la gestión de la pedagogía se desarrolla a través de iniciativas voluntarias de

12 Sin embargo, sus resultados en los exámenes ministeriales han mejorado notablemente a lo largo de los años con la puesta en marcha de la GBR. Su carácter desfavorecido está también asociado a una fuerte proporción de estudiantes con necesidades educativas especiales. 
la dirección sobre el seguimiento de los años de estudio problemáticos o de grupos de docentes por disciplina sobre la base de estadísticas disponibles. Esta estrategia se desarrolla, sin embargo, de manera fuertemente participativa, estando atenta la dirección a que, más allá del obstáculo, la GBR se transforme en una «oportunidad para el equipo-centro». La dirección desarrolla para ello una lógica de la acción que interviene en la zona de jurisdicción y de autonomía del equipo docente, pero con su participación y un consentimiento relativo. La lógica es bastante similar a la del Centro de las Cataratas, con una diferencia: los resultados son, desde el principio, mejores y el centro goza de una situación de mayor privilegio.

En estos dos centros todo funciona como si una estrategia de gestión de la pedagogía bastante ofensiva hubiera sido legitimada a través de una estrategia colaborativa y participativa de las direcciones que han logrado apaciguar la resistencia sindical. De ello resulta al final un profesorado que acepta, al menos parcialmente, la puesta en marcha de la GBR y las limitaciones a su autonomía de decisión o la insistencia sobre los resultados y la reflexividad performativa.

\section{Conclusión}

Hemos mostrado que la puesta en marcha de la GBR por parte de la comisiones escolares y los centros educativos se acompaña de diversas estrategias que, según apuntan, hacen evolucionar las prácticas y las identidades profesionales docentes. El profesorado está sometido a una obligación (normativa) de reflexividad sobre la eficacidad de sus prácticas. Paralelamente, las direcciones de los centros buscan, de manera más o menos directiva o participativa, «armonizar» las prácticas docentes en lo que respecta la cobertura de los programas y la evaluación del alumnado, para hacerlo más conforme y mejorar el «rendimiento» en relación a las evaluaciones externas del ministerio. Para ello, las direcciones se apoyan en medios de presión basados en las prescripciones legales o las comparaciones de resultados académicos. Así, la GBR se acompaña de diversas estrategias de gestión de las prácticas pedagógicas del profesorado, que tienden, por una parte, a incidir sobre su autonomía de decisión (en el plano de las prácticas de cobertura de los programas y de la evaluación), y a orientar, en mayor medida, su reflexividad profesional sobre la cuestión de la eficacia de sus prácticas. En este sentido, se ve que la preponderancia del poder de gestión en el centro se acompaña de una adecuación, de una reducción, y de un control fuerte de la autonomía del profesorado en materia de cobertura curricular y de prácticas de evaluación. Esto confirma numerosos estudios sobre las prácticas de accountability «duro» que han mostrado que tienden a generar formas de reducción curricular, de teaching to the test, pero también una forma de estandarización de las prácticas en función de su efecto inmediato sobre los resultados a las evaluaciones externas (Mons, 2009 ; Rozenwajn y Dumay, 2014). Sin embargo, las prácticas y relaciones pedagógicas en la clase siguen estando relativamente fuera de esta tendencia y no son afectadas más que indirectamente, a través de la promoción de una reflexividad muy orientada solamente por la cuestión de los resultados escolares.

Las respuestas del profesorado a estas demandas se construyen de manera compleja y variada, en función del sentido que da a las demandas que les son hechas. Dos forma de respuesta mayores (aceptación relativa u oposición relativa a las demandas institucionales) cohabitan con una respuesta menor, no tematizada por Coburn: la renuncia del profesorado a una práctica. Frente a las demandas relativas a la cobertura de los programas o a las prácticas de evaluación, o a la obligación de reflexividad sobre la eficacia de sus prácticas, la aceptación relativa significa que el profesorado tienden a ajustar sus prácticas a estas demandas sin cambiar sus sistemas de valores y de representaciones (asimilación) porque hay, de hecho, congruencia parcial entre estas últimas y dichas demandas institucionales. Más raramente, el profesorado 
señala que sus prácticas y sus maneras de ver estas demandas han evolucionado en efecto (acomodación). Por el contrario, algunas oposiciones relativas a estas demandas son también observables bajo la forma, ya sea de rechazo, ya de respuestas simbólicas/disociación. Esto concierne, en particular, a la demanda de ajuste del nivel de exigencias de las prácticas de evaluación. Hemos visto también que, cuando las presiones de la dirección son fuertes y controlables por instrumentos de seguimiento (fundamentalmente estadísticos), el profesorado puede conformarse, a su pesar, a tal o cual demanda institucional (sobre todo de reducción del currículo enseñado). Dicho de otro modo, el profesorado renuncia a una práctica sin cambiar, por ello, sus marcos cognitivos y normativos, lo que sucede no sin tensiones identitarias.

Hemos destacado también que las frecuencias de las respuestas varían en función del centro. El análisis de las razones de estas variaciones debe explorarse más. Una constelación de factores parece efectivamente en interacción. Más allá de la incidencia del contexto (historia, población estudiantil, resultados del centro, expectativas de la CE), del ethos y de la identidad de los actores (fundamentalmente docentes), la dinámica y la configuración de las relaciones entre dirección y profesorado podría ser, a la vez, fuente y resultado del sentido conferido a la GBR, sobre todo del lado del profesorado. Dicho de otro modo, proponemos la hipótesis según la cual las respuestas del profesorado no se deben solamente a la relación entre los valores y creencias individuales, de una parte, y presiones institucionales de la otra, como lo tematiza Cynthia Cobrun. Estas respuestas podrían depender también de las configuraciones entre las relaciones entre actores, la dinámica y la dialéctica entre estrategias de la dirección del centro y las respuestas del profesorado. Efectivamente, en dos centros observados (Centros de la CE del Norte, esto es, Centro de los Suburbios y Centro de las Cataratas), parece haber una forma de círculo virtuoso entre las estrategias participativas de la dirección y la adhesión relativa del profesorado a la GBR, lo que ha favorecido la capacidad de la dirección de los centros investigados de desarrollar una gestión de la pedagogía bastante intervencionista. Por contra, en los otros dos centros (Centro de las Montañas y Centro de los Campos), una configuración de actores relativamente tensa, incluso conflictual, da lugar a estrategias variables de la dirección, frente a los desacuerdos manifestados por el profesorado. La imposición de la GBR sobre las prácticas docentes parece también más limitada.

\section{Referencias bibliográficas}

Abbott, Andrew (1988). The system of professions. An essay on the division of expert labor. Chicago : The University of Chicago Press.

Bernstein, Basil (1977). Class, codes and control. Volume 3. Towards a theory of educational transmissions. London: Routledge and Kegan Paul.

Champy, Florent (2009). La sociologie des professions. Paris: Presses universitaires de France.

Coburn, Cynthia (2004): "Beyond Decoupling: Rethinking the Relationship Between the Institutional Environment and the Classroom". Sociology of Education, 77 (3), 211-244. doi: 10.1177/003804070407700302

Crahay, Marcel (Dir.) (2012). L'école peut-elle être juste et efficace? De l'égalité des chances à l'égalité des acquis. (2éd.). Bruxelles: de Boeck.

Freidson, Eliot (2001). Professionalism. The third logic. Cambridge: Polity

Fullan, Michael (2007). The new meaning of educational change. New-York et Londres: Routledge. 
Lang, Vincent (1999). La professionnalisation des enseignants. Sens et enjeux d'une politique institutionnelle. Paris: PUF.

Maroy, Christian (2010): “Contextes, niveaux et médiations dans une recherche comparative internationale. Quelques réflexions à partir de la recherche européenne Reguleduc”. Education Comparée, 2, 143-168.

Maroy, Christian; Brassard, André; Mathou, Cécile; Vaillancourt, Samuel y Voisin, Annelise (2016). "La mise en œuvre de la politique de gestion axée sur les résultats dans les commissions scolaires au Québec. Médiations et mécanismes d'institutionnalisation d'une nouvelle gestion de la pédagogie". Rapport de recherche, Université de Montréal, Montréal.

Maroy, Christian; Brassard, André; Mathou, Cécile; Vaillancourt, Samuel y Voisin, Annelise (2017): “La Co-construction de la gestion axée sur les resultats: les logiques de médiation des commissions scolaires". Revue des sciences de l'éducation de McGill, à paraitre.

Maroy, Christian (2017). "La nouvelle gestion publique en éducation au Québec: vers une gestion de la pédagogie". Sociologie du Travail, 59 (4).

Meyer, John. W.; Rowan, Brian. (1977): "Institutionalized organizations: Formal structure as myth and ceremony". American journal of sociology, 83 (2), 340-363.

Mons, Nathalie (2009). "Effets théoriques et réels des politiques d'évaluation standardisée". Revue Française de Pédagogie, (169), 99-139.

Ranson, Stewart (2003): "Public accountability in the age of neo-liberal governance". Journal of Education Policy, 18 (5), 459-480. doi: 10.1080/0268093032000124848

Rozenwajn, Esteban y Dumay, Xavier (2014): “Les effets de l'évaluation externe sur les pratiques enseignantes: une revue de la littérature". Revue Française de Pédagogie, (189), 105-137.

Spillane, James P. (2004). Standard deviation: How schools misunderstand education policy. Cambridge: Harvard University Press.

Spillane, James P.; Diamond, John B.; Burch, Patricia; Hallett, Tim; Jita, Loyiso y Zoltners, Jennifer (2002): "Managing in the Middle: School Leaders and the Enactment of Accountability Policy". Educational Policy, 16 (5), 731-762. doi: 10.1177/089590402237311

Spillane, James P.; Reiser, Brian J. y Gomez, Louis M. (2006): "Policy implementation and Cognition. The role of human, social, and distributed cognition in framing policy implementation" en M. I. Honig (dir.). New directions in education policy implementation. Confronting complexity. Albany: State University of New York Press.

Tardif, Maurice y Lessard, Claude (2002): Le travail enseignant. Bruxelles: De Boeck Universités. 


\section{Notas biográficas}

Christian Maroy es sociólogo y catedrático de la Facultad de Educación de la Universidad de Montreal. Es titular de la Cátedra de Investigación de Canadá en Políticas Educativas. También es investigador asociado en GIRSEF, Universidad Católica de Lovaina. Sus intereses de investigación se centran en políticas educativas y nuevas formas de regulación de la educación, sus efectos sobre las desigualdades escolares y los cambios en la profesión docente.

Cécile Mathou es estudiante de doctorado en Educación Comparada y Fundamentos de Educación en la Facultad de Educación de la Universidad de Montréal y miembro del equipo de investigación de la Cátedra de Investigación de Canadá en Políticas Educativas.

Samuel Vaillancourt es investigador profesional de la Cátedra de Investigación de Canadá en Políticas Educativas. 